\title{
Security for e-Advertisements using Digital Watermarking and Mobile Agents
}

\author{
Roopa G $\mathrm{M}^{1}$, and Dr Nirmala $\mathrm{C} \mathrm{R}^{2}$
}

\begin{abstract}
The growth in the Internet and high speed communication networks has explored the means of new eBusiness, entertainment, content delivery at run-time, online transactions, personal communications, web magazines/newspapers, e-Publishing/e-Advertising and many more. Such Online Businesses requires a technique to protect intellectual property rights for the digital content that is shared in a distributed environment which usually leads to the issues related to data security. Thus to resolve the problems associated in the field of Electronic copyright protection and data security, Digital Watermarking acts as the core technology. In our previous work, we have proposed a hybrid framework for e-Advertising applications to post the advertisements at various ad-publisher sites. The framework introduces the concept of aggregating the advertisements from set of advertisers, which are later published simultaneously. This makes use of concurrent Mobile agents to process the advertiser request in parallel. In this research work, we propose a Digital Watermark Detection system for e-Advertising applications, which adopts Concurrent Mobile Agents to manage and protect the advertisements (Images + Text) from illegal usage by providing 2-stage copyright protection scheme before publishing at various ad-Publisher sites. With the use of Concurrent Mobile Agents the copyright detection is greatly improved. The proposed work is implemented using Aglet Software Development Kit (ASDK-2.0.2) which is a Java based Mobile Agent platform and MySQL for storage purpose.
\end{abstract}

Keywords - Advertisements, Copyright Protection, Digital Watermark, e-Advertising, Mobile Agents.

\section{INTRODUCTION}

In this recent year, the rapid growth in the multimedia data has got more and more popularity due to the huge growth of Internet, which has provided an easy means for the reproduction and communication of such large volume of data. And today millions of web and Mobile users are benefited from that advancement of such simple and fast digital data exchange. Such benefits come together in hand with problems and threats associated with ensuring digital copyright protection, proof of authenticity and contentoriginality verification as an essential requirement for online dissemination of sensitive and specialized, format, legal, financial and religious content [1]. All such multimedia components on the Internet are classified into images, text,

\footnotetext{
${ }^{1}$ Research Scholar, Department of CS\&E, BIET- Davangere-577004.

${ }^{2}$ Prof. \& Head, Department of CS\&E, BIET-Davangere-577004.
}

video, audio and ensuring security for such media type is a challenging task.

Much of the research work has proved that, digital watermark provide a promising solution to deal with problem of data security and information misuse. The traditional design method used to embed the watermark for the digital data adopts the Client/Server approach. Here, the well understood relation between the advertisers and the publishers/servers needs to have a common one-to-one relation as in case of e-Commerce transactions that typically involves hashing or encryption algorithms being distributed between two or more parties [5]. Furthermore, the use of private keys for each Client/Server needs to be generated separately which leads to the problem of overloading the server as the number of requests increases from the client side and also over occupy the network bandwidth. This also needs a reliable connection between the Client/Server until the request is processed.

The advancement in the Internet Technology has revamped the lives of millions of users around the world, where eAdvertising forms a new trend in the Business which plays a very important role for majority of web users by providing them the platform to buy and sell their products/items. This wide variety of product/items information sharing among the web users has introduced the problem of data security and information misuse of the advertisements posted by set of advertisers. Thus the copyright owner of the advertisements (image + text) should find a way for protecting their product information like image, cost, model, make, description etc, against the illegal usage of the advertisement information.

In order to overcome the above issues Mobile Agents are used, which are treated as an alternative for the Client/Server model. They are the emerging technology which makes it easy to design, implement and maintain distributed applications [2].It is observed that Mobile Agents reduce network traffic, provide an efficient means of overcoming network latency and their ability to operate asynchronously and autonomously of the process that created them [3].

The proposed work deals with e-Advertising framework which combines watermark detection technique and Mobile Agents, which adopts distributed processing and checks for the digital watermark directly on the advertisements at various ad-publisher sites. The framework deals with collecting, aggregating and loading the advertisements 
request from various advertisers to post on different adpublisher sites. The distribution of such electronic product/item information creates serious security issues even if there exists a trusted network. One solution is to deal with security issues and the problem is that every advertiser must embed permanently a copyright policy watermark to the advertisements, to avoid illegal usage of the product information. But it is observed that most of the advertisers are careless and ignorance due to the curiosity of posting the advertisements due to the competition in the open market for buying and selling their products. In such scenario, we provide a two stage copyright policy scheme for detection of watermark applied for the set of advertisements. One during the advertisements creation level and the second one at the Copyright Management Agency (CMA) level..

\section{MOTIVATION OF THE PROPOSED WORK}

As millions of websites are flooding the Internet, it might seem easy for the illegal usage of the information which might be copied from one website to another without the permission of the owner. The main motivation is that it is required or even desirable that particular (product/item information) sensitive content should be freely published at multiple ad-publishers sites for wider outreach without any information loss or illegal usage of the copyright information.

\section{RELATED WORK}

Ziad Al Bkhetam and Nawar Al Awa [1] proposed a complete system for watermarking and web tracing of color images by adopting blind detection model that depends on SIFT algorithm to improve the system tracing performance. Their work traces the images on the web using watermark Mobile Agents. They have shown that this work is efficient in dealing with large-size images across congested network.

Fu-Cheng You et. al. [2] have identified the drawbacks of the conventional method of digital copyright protection. They put forward a new digital watermarking tracking system based on Mobile Agent paradigm, which authenticates the digital content directly on the remote mainframe by using the distribution and Mobile characters of Mobile Agents. The efficiency of the copyright protecting and tracking is greatly improved by adopting the Mobile agent technology.

Nagaraj V Dharwadkar and B B Amberker [3] proposed Digital Rights Management system for online digital image library copyright protection based on watermark tracing. The tracking of watermark on the remote hosts machines is done using active Mobile Agents which supports the coordination of several component tasks across distributed and flexible networks of information services.

LIU Quan and LIU Hong [4] have suggested that the integration of Mobile Agents and Digital Watermark has been intensively used in the recent years. A framework model has been put forward to design copyright protection based on digital watermark and Mobile Agents. This framework reduces the network load as the watermark detection is carried at the remote hosts.

Omar Tayan et. al. [5] have addressed the problems and threats associated with verification of data integrity, proof of authentication, tamper detection and copyright protection for digital-text content. They proposed a hybrid approach based on zero-watermarking and digital signature like manipulations for sensitive text documents in order to achieve content originality and integrity verification. Enhancements in resource utilization and reduced redundancies were achieved in comparison to traditional encryption based approaches.

Jian Zhao and Chenghui Luo [6] present a complete Digital watermark agent system that triggers an agency to dispatch digital watermark Agents to agent servers to perform various tasks on the server. Their work was the first Mobile computing approach towards enforcement of digital watermark. This contributes to the fundamental knowledge in the areas of digital security, intelligent agents and collaboration technology

\section{SySTEM ARChITECTURE}

The scenario of the copyright protection system which combines Digital Watermarking technique and Mobile Agent paradigm is given in Fig. 1.

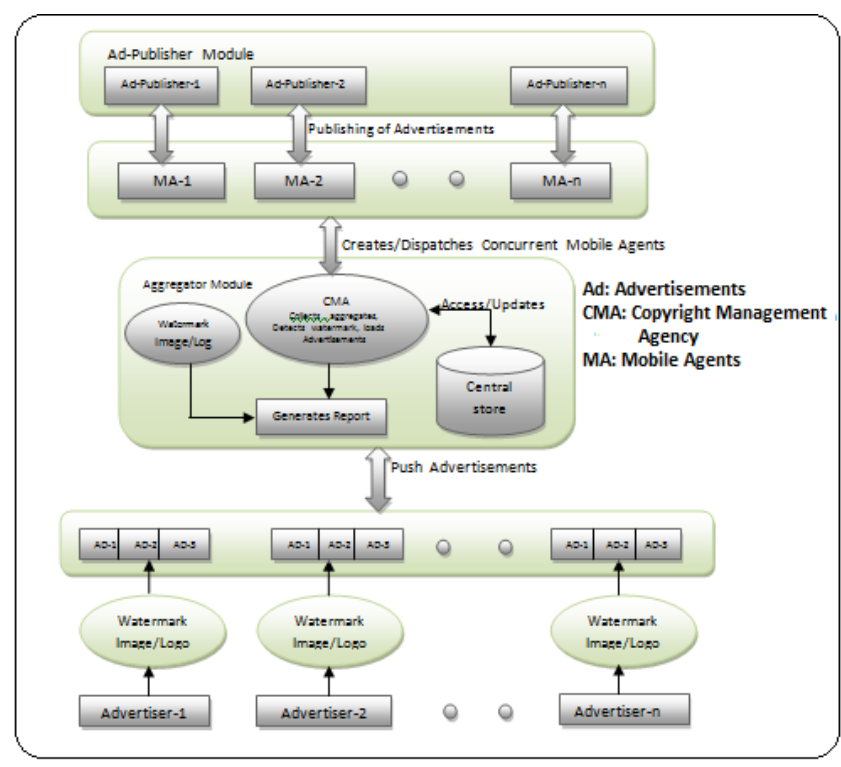

The proposed framework includes the following modules Advertiser Module, Aggregator Module and ad-Publisher Module:

\section{Advertiser Module (Advertisement Owner):}

Advertiser creates the advertisements (image +text) and has all the rights on the set of advertisements. Every advertiser embeds watermark on the advertisements by applying watermarking embedding algorithm, which stamps the symbol of copyright on all the product files to protect against unauthorized use. The owner maintains a database to store the legal information of the product/item like year of make, model, cost , description, image etc.. This information 
may be required for the later use while tracking the advertisements before publishing at various ad-publisher sites.

The stationary agent in this module loads the watermark embedded advertisements to the Mobile Agent which are pushed to the aggregator module for publishing at various adpublisher sites and waits for the acknowledgement from the aggregator module.

The pseudocode for embedding the watermark: class watermarkimage \{ public void main ( ) \{ retrieve the path for advertisements(image+text) create a bufferedimage object for every advertisement initializes the necessary graphics properties create graphics object and add the original file to it set the font for the watermark text set the unicode character add the watermark text and set the status code new watermarked advertisement files are created successfully

\}

\}

\section{Aggregator Module:}

This module provides an environment to detect the watermark applied on the advertisements. It also provides the Mobile Agent navigation services to publish the advertisements at various ad-publisher sites simultaneously. This module includes the following sub-modules:

- Central Store: The server maintains the information of different advertisers like, the author watermark embedded, legal product/items information. This information helps in building the knowledgebase about different advertisers. It also generates the report after detecting the advertisements for watermark. This report can be used for taking certain decisions.

- Copyright Management Agency (CMA): CMA is a stationary agent which plays an important functional part and acts as the Watermark Agency which performs the set of operations like advertiser and ad-publisher management, collecting, aggregating and loading the advertisements, watermark detection, report generation, embedding watermarking, dispatching Digital Watermark Agents to various predefined ad-publisher sites.

The embedding process starts with inserting a unique image/logo/text as the owners right to the advertisements which is applied locally by the advertisers. On completion of the watermark embedding process, every advertiser submits the product/item information and requests the CMA for publishing the advertisements at various ad-publisher sites. This agent collects aggregates and performs the task of detecting the watermarks applied for the advertisements. Next, generates the report and categorizes the list into watermarked and un-watermarked advertisements, then selects only those advertisements which are unmarked and embeds the copyright protection. It also updates the central store after processing the generated report. Finally, CMA prepares and provides the guidelines for execution of concurrent Mobile Agents suitcase which contains the destination (predefined ad-publisher sites), termination condition (after publishing), action policies (for illegal usage of the information) and advertiser/aggregator watermark details.

The stationary agent in CMA has the following functions:

- Receiving request from every advertiser.

- Collecting, aggregating and processing the advertisements from various advertisers.

- Checks the advertisements for copyright protection by the advertiser.

- Generates the report by categorizing the set of advertisements into watermarked and un-watermarked.

- Process the report and embeds the watermark for unwatermarked advertisements with an ADMIN image/logo/text.

- Updates the central store after processing.

- Provides an environment for the execution of Concurrent Mobile Agents.

- Creates, Loads and Dispatch concurrent Mobile Agents to various ad-publisher sites for publishing the advertisements.

- Waits for acknowledgement after successfully publishing the advertisements.

Pseudo code for the CMA Stationary Agent: CMA Stationary Agent

//Class for CMA Stationary Agent at the Aggregator Module import *.aglet;

class Agency extends Aglet \{

public void run( ) \&

Agency Aglet( object init) \{ listen for arrival of Mobile Agents from the advertise sites on arrival of advertiser Mobile Agents

AgencyResponse(advertisement)

aggregates only the successfully received advertisements

Detectiontask(aggregated_advertisements)

processreport(unmarked_advertisements)

creates concurrent Mobile Agents to reach various adpublisher sites loads Mobile Agent with watermarked advertisements \& dispatch

get_proxy $($ Aglet Id $)$ \{

Dispatch ( )!

waits for the acknowledgement

\}

$$
\text { j }
$$

public void AgencyResponse( reqAdvertisementType advertisement) \{

for every advertiser in the advertiser list

if Agency accepts and process the request successfully

collects and aggregate the advertisements

send acknowledgement to the advertiser site

else 


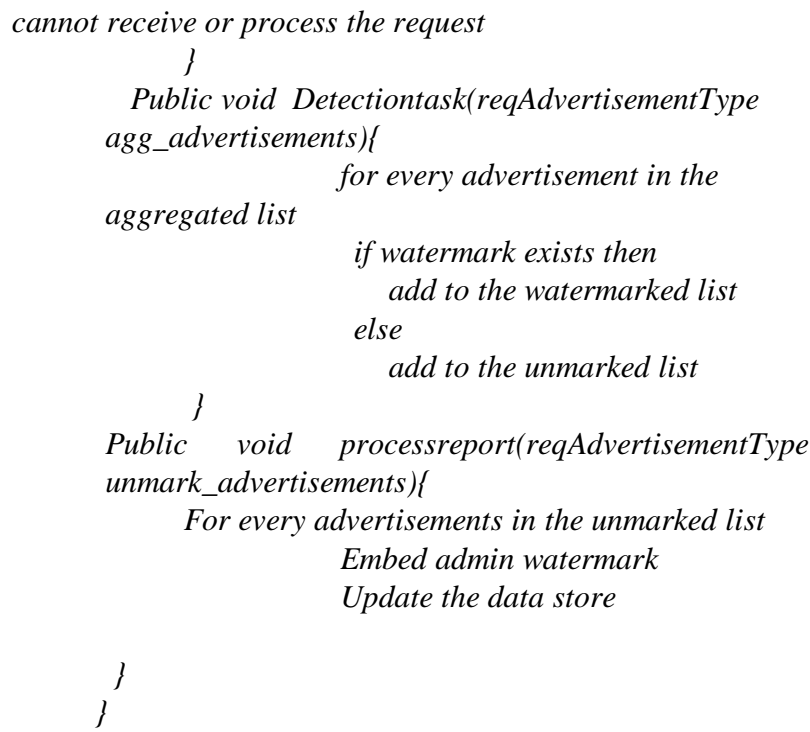

\section{ad-Publisher Module:}

This module acts as publisher websites which distribute media content online or offline. They offer digital content download and only few might take some precautionary measures to avoid unauthorized or illegal usage of the product/item information.

\section{CONCLUSION}

Digital Watermarking is undoubtedly the best way of protecting various forms of digital contents in a distributed environment. As e-Advertising being the part of e-Commerce applications, it requires that copyright protection which is necessary to avoid illegal usage of the product/item information which are mounted to majority of web users. However, it is observed that only few of the advertisers apply efficient techniques to protect their advertisements and most of the advertisers ignore to protect due to the curiosity of posting the advertisements as there is a high competition in the open market for buying and selling their products. In this work, we put forward a framework to show the relationship between e-Advertising applications and Digital Watermarking technique which adopts Mobile Agent paradigm for copyright detection on advertisements. Here, we provide 2-stage copyright protection scheme on the advertisements one during the advertisement creation level and second at the Copyright Management Agency level from illegal usage. Thus protecting the intellectual property of the product/item information in a distributed environment can be automatically applied by adopting the Mobile Agent paradigm. Further more Mobile Agent paradigm overcomes the drawbacks of the existing approach used for digital watermarking.

\section{FuTURE SCOPE}

The future work focuses on tracking the advertisements , where Concurrent Mobile Agents can be assigned the task of tracking the advertisements after being published at various pre-defined ad-publisher sites. Mobile Agent at every adpublisher site matches the security or copyright policy embedded by the owner of the content after publishing. If any mismatch occurs, then those advertisements can be blocked from publishing or it can generate the report and request the stationary agent in the CMA to take necessary actions. Next, by adopting the mobility and parallelism characteristics of Mobile Agents, the task of watermark tracking on advertisements at various ad-publisher sites can be faster and efficient.

\section{ACKNOWLEDGEMENTS}

It is great pleasure to express my deep feeling of gratitude to my respected Supervisor, Prof. Dr. Nirmala C R for her great encouragement, constant support, valuable and expert guidance which provided desired moral and confidence to carry out this research work. I take this opportunity to thank my Institute for providing me the facilities to carry out my research. I am also thankful to my colleagues for their direct and indirect help in completion of this research work.

\section{REFERENCES}

[1] Ziad Al Bkhetan and Nawar Al Awa "Robust Color Image watermarking and Web-Tracing System Using Digital Wavelet Transform and Mobile Agents". In the Proceedings of International Arab Journal of $e$ Technology. Vol.3, No.2, June 2013.

[2] FU-Cheng You, Yang Li,Xu-Yan Tu "Research on Mobile Agent-Based Digital Watermark Tracking System and Communication Security" Proceddings of the 2007 International Conference on Wavelet Analysis and Pattern Recognition,Beijing , China 2-4, Nov-2007.

[3] Nagaraj V Dharwadkar and B B Amberker "Digital Watermark Tracking using Intelligent Multi-Agents System" Journal of Applied Computer Science and Mathematics.N0.7- Special Issue.

[4] LIU Quan and LIU Hong "Application of Digital Watermark and Mobile Agent in Copyright Protection System" International Conference on Computer Science and Information Technolory, 2008. https://doi.org/10.1109/iccsit.2008.35

[5] Omar Tayan, MuhammadN. Kabir and Yaseer M. Alginahi "A Hybrid Digital-Signature and Zero-Watermarking Approach for Authentication and Protection of Sensitive Electronic Documents" Hindawi Publishing Corporation, The Scientific World Journal,Volume 2014, Article Id 514652,2014 . https://doi.org/10.1155/2014/514652

[6] Jian Zhao and Chenghui Luo "Digital Watermark Mobile Agents" In the Proceedings of NISSC'99, Arlington, Virginia, Oct 18-21,1999, 138-146.

[7] S H Kwok, C C Yang and K Y Tam "Intellectual Property Protection for Electronic Commerce Applications" Journal of Electronic Commerce Research, Vol.5, No.1, 2004 